\title{
Sistemas de Información Electrónica al Servicio de la Investigación
}

\author{
Ignacia Robledillo Izquierdo \\ Chadwyck-Healey España
}

\subsection{Resumen}

La objetivo de esta comunicación es presentarles, de forma breve, la evolución en el tiempo, desde la información escrita (en soporte papel) hacia los nuevos soportes electrónicos, examinando al mismo tiempo como esto ha beneficiado a los investigadores, y como las bibliotecas y editoriales, en concreto la editorial Chadwyck-Healey, han tenido que ir adaptando sus servicios y productos a los nuevos tiempos y demandas del investigador y en general de los usuarios finales de información.

Partiendo de esta base, la primera pregunta que debemos plantearnos es: ¿qué es la información electrónica?. Desde luego la información electrónica no es nada nuevo, hemos recibido información electrónica durante años a través de la televisión y la radio. Pero centrándonos en el área que nos interesa, en el mundo académico y de la investigación, únicamente nos interesa la información electrónica digital, es decir información que pueda ser manejada por ordenadores. Por supuesto, es importante comenzar hablando de los "precursores" de los soportes digitales y de su importancia para el acceso a la información. Así comenzaremos por la reproducción de información en Microfichas, hasta llegar a los actuales soportes electrónicos (Autor).

Palabras claves: Sistemas de información digitales. Microfilmación.

\subsection{Abstract}

The aim of this communication is to make a brief presentation of the evolution from the written documents (paper format) to the new electronics information sources, looking at the same time into the benefits for researchers in any scientific field. It is also interesting to understand how the libraries and publishers companies, particularly Chadwyck-Healey, have changed their products and services to answer the end-user needs. 
If we ask ourselves what electronic information is, we will realise that it is nothing new. We have received it for years over television and radio. But electronic information we are talking about, related to the academic and research world, is digital information, that is, in the form that computers can handle. It is important to begin talking about the "predecessors" of the actual digital formats and its importance for accessing information resources. So we will begin talking about the evolution of the microfiche to the new electronic formats. (Author)

Keywords: Digital Information systems. Microforms.

\section{Microficha}

Antes de que aparezca el soporte de la Microficha, la investigación es costosa y lenta, pero lo peor es que el intercambio entre investigadores y entre grupos apartados de demandantes de información es muy escaso, principalmente porque las fuentes primarias de información, el documento en sí, está muy distribuido geográficamente y es de difícil acceso. Esto tiene como principal consecuencia que existan pocas herramientas documentales al servicio del investigador y de cualquier demandante de información. Con la reproducción de la información en soporte microficha se van a conseguir fundamentalmente dos cosas:

- Evitar el deterioro del documento primario.

- Facilitar la distribución y uso de la información contenida en los mismos.

El investigador se encuentra con que puede consultar información bibliográfica de carácter general, así como especializada, de una forma mucho más rápida y accesible.

Precisamente, esta es la base sobre la que Chadwyck-Healey comienza su actividad hace aproximadamente 25 años, adquiriendo los derechos de edición, en microficha, de catálogos de grandes Bibliotecas, que a partir de entonces estarán al alcance de un público mucho mayor y diseminado "geográficamente". En concreto podemos citar el "Catálogo General de Libros Impresos de la Biblioteca Nacional Española" o "Bibliothèque Nationales: The Author Catalogues of Printed Books", con 4.982 y 4675 microfichas respectivamente.

Por otra parte, también comienza a difundirse información algo más especializada y de interés para grupos concretos y usuarios con necesidades de información diferentes. Es el caso de colecciones como "Catálogo de la colección Gómez-Imaz", que reproduce en microficha un total de 5.000 trabajos impresos y manuscritos relacionados con la Guerra de la Independencia Española (18081814).

La reproducción en formato microficha facilitará el ahorro en costes de almacenaje, se ganará también en rapidez de acceso y en accesibilidad a las fuentes de 
información. Sin embargo este es un formato costoso, en cuanto a la producción del mismo, y la propia evolución de la tecnología nos llevará a lo que supone el siguiente salto en el uso y producción de herramientas de referencia al servicio de la investigación: el CD-ROM.

\section{CD-ROM}

Este nuevo soporte supone el primer cambio tecnológico global tanto a nivel de software y hardware, como de contenidos. Podremos comenzar a hablar de información electrónica, información digital tal y como hoy la conocemos, de gran utilidad y con enormes ventajas para la investigación y la referencia.

El soporte CD-ROM otorga a la investigación y al trabajo académico una velocidad y flexibilidad que hoy ya nadie duda; haciendo posible una mayor capacidad de almacenaje, permitiendo mayor rapidez en el tratamiento y la búsqueda de la información; permitiendo el uso de la información por un número mayor de usuarios; y aumentando el rendimiento y la eficacia del propio trabajo individual. En este punto, a nadie se le escapan las ventajas que supone el poder conocer, en pocos segundos, que obras de un determinado autor podemos encontrar en una biblioteca concreta, que se ha escrito en los últimos años sobre el área de investigación que nos interesa, etc.

En un primer momento, los contenidos que pasan a este nuevo formato son similares a los que se venían reproduciendo sobre soporte microficha, y que ahora, sobre CD-ROM, supondrán tanto un ahorro en costes de adquisición, al ser éste un soporte mucho más barato de producir, como un ahorro en costes indirectos.

Un ejemplo claro de esto lo tenemos en las Bibliografías Nacionales en CDROM, que por una parte permiten a cualquier usuario localizar rápidamente información sobre autores o temas de su interés, así como conocer la producción editorial de cada país. Y que por otra parte, facilitan enormemente el trabajo técnico dentro de las propias bibliotecas, agilizando y facilitando los costes de catalogación (retrospectiva o corriente).

Actualmente Chadwyck-Healey distribuye en CD-ROM un total de seis Bibliografías Nacionales europeas, así como tres grandes catálogos de Bibliotecas Nacionales (Inglaterra, Francia y Florencia).

A partir de aquí, y de mano del CD-ROM, comenzará a producirse la auténtica revolución en el mundo de la investigación, con una demanda real, y por consiguiente con la aparición de obras más especializadas, destinadas a campos específicos del conocimiento, o catálogos colectivos de diferentes bibliotecas o instituciones. Es el caso de bases de datos como Bibliotecas sin Fronteras, Novum Regestrum o DocThèses por poner un ejemplo. La primera de ellas es un

Scire. 3 : 2 (jul.-dic. 1997) 107-113 
catálogo colectivo de fondos iberoamericanos existentes en bibliotecas españolas; Novum Regestrum es un catálogo de fondo antiguo, de los siglos XV-XIX, de la Asociación de Bibliotecas Nacionales de Iberoamérica (ABINIA); y por último DocThèses, es un catálogo de más de 300.000 tesis (sobre cualquier disciplina académica) leídas en Universidades francesas desde 1972 hasta nuestros días.

Como vemos el contenido de las bases de datos empieza en a ser muy variado y se ajusta a necesidades cada vez más específicas, con lo que el investigador y cualquier usuario final de información empieza, como paso lógico, a demandar la localización de los fondos a los que ahora accede en formato electrónico, y que ya aparece en muchas de las bases de datos en CD-ROM. Esto junto con una mayor complejidad y especialización en los temas, será el inicio de un intercambio de conocimientos y experiencias entre investigadores a nivel nacional e internacional que llevará al desarrollo de nuevas tecnologías y formas de comunicación como, por ejemplo, la red Arpanet en Estados Unidos, que surge en los años setenta, se desarrolla durante los ochenta y terminará siendo la base para lo que hoy conocemos como Internet.

En este punto, es importante destacar la importancia de un tema como es el interfaz de los nuevos soportes electrónicos. Se hace evidente la necesidad de acercar la información a los usuarios mediante interfaces amigables e intuitivos, que no hagan necesario un aprendizaje complejo para llegar a la información que es lo que realmente interesa. En ese sentido, las actuales bases de datos en CDROM y por supuesto las que son accesibles a través de Internet, han permitido transformar los ordenadores y las información electrónica en potentes herramientas de almacenamiento y difusión de información, para cualquier tipo de usuario.

Así en los años 90, el CD-ROM ya está establecido como una herramienta de referencia básica para la investigación en todas las áreas, tanto en Ciencias como en Humanidades y las bases de datos cada vez son más ricas en contenido con una tendencia, cada vez mayor, al texto completo. Es decir las necesidades de información de los investigadores se cubren con fuentes de referencia que tienden hacia tres puntos claves:

- Mayor cantidad de información.

- Mayor calidad de información.

- Mejor acceso al documento final.

Chadwyck-Healey, como editorial electrónica, ha ido reflejando en sus publicaciones las necesidades y demandas de los usuarios, disponiendo actualmente de un amplio catálogo de bases de datos, tanto referenciales como a texto completo, enfocadas al mercado académico y bibliotecario del mundo entero; así podemos destacar Autoridades de la Biblioteca Nacional Española de gran ayu- 
da para el trabajo técnico en bibliotecas, Teatro Español del Siglo de Oro o Patrología Latina, obras de texto completo, que permiten trabajar sobre reproducciones exactas de los documentos fuente, o International Index to Music Periodicals, que nos presenta una obra de referencia internacional para localizar publicaciones periódicas especializadas en el mundo de la música.

\section{Internet}

Con el desarrollo de la red Internet, también conocida como la "Aldea global", desaparecerán las barreras geográficas, culturales, y políticas, favoreciendo un grado de comunicación, cooperación, acceso a las fuentes de información y colaboración, no conocido hasta el momento.

La información electrónica empieza a llegar al usuario final en nuevos soportes y con nuevos formatos; los sistemas hipertexto e hipermedia se generalizan y están en la base del éxito generalizado de Internet. El World Wide Web nace para ayudar a la investigación académica. Sin embargo, también nos encontramos con los problemas que estos nuevos sistemas pueden provocar, como son la saturación de fuentes de información y de datos que pueden producir en el usuario una desorientación.

La propia comunidad académica comienza a demandar nuevos servicios o productos editoriales entre los que destacan aquellos que ayuden a orientarse en Internet, que "reúnan" a usuarios con intereses comunes, y que faciliten un servicio de valor añadido. Todo esto junto con la facilidad en el acceso y el mantenimiento de los nuevos productos, así como un abaratamiento de costes.

En cuanto al coste del acceso a la información electrónica, es fundamental tener en cuenta el ahorro en tiempo, personal, desplazamientos, etc. del que se beneficia una organización, cuando sus investigadores pueden, por ejemplo:

- localizar en tiempo récord fuentes bibliográficas,

- leer resúmenes de artículos para descartar directamente los que no sean de su interés,

- saber a que institución dirigirse para localizar el documento primario,

- comparar versiones de un mismo texto, separadas en el tiempo,

- acceder al texto completo del documento que necesita.

La entrada de Chadwyck-Healey en Internet, se inicia con la base de datos Periodicals Contents Index (PCI) y continúa con el servicio Literature Online.

PCI es una base de datos fundamental para la investigación retrospectiva en Humanidades. Actualmente recoge 6 millones de artículos, que ocupan 10 CDROMs, y que continúa creciendo. Recoge los índices de contenido de las publi 


\section{2}

caciones de Humanidades, que han sido consideradas como de mayor relevancia a nivel internacional, desde el siglo XIX hasta el año 1990.

En cuanto a "Literature Online" Chadwyck-Healey ha creado un servicio totalmente enfocado - por ahora - al ámbito de la Filología Inglesa. El investigador llama a una URL donde puede establecer una serie de criterios o palabras claves para filtrar obras a texto completo: poemas, drama, ficción, etc. Actualmente se recogen unas 100.000 obras en total, de las que 80.000 son fondos de Chadwyck-Healey. Una vez en el texto completo, se puede saltar a otras obras del autor, a su biografía, a otros sites que hablen del tema en cuestión o incluso consultar una palabra en un diccionario que contextualice el significado del término en su época.

Con Literature Online, Chadwyck-Healey está consiguiendo dos cosas fundamentales:

- Volcar todos nuestros productos a texto completo (que ya estaban en soporte CD-ROM), para así poder cruzarlos desde un mismo interfaz.

- Crear un punto de reunión "virtual" de la comunidad científica con intereses especializados en este tipo de fondos, que además encontrarán un valor añadido, al encontrar aquí otra información su interés y presente en Internet.

Algo a destacar de estos nuevos servicios en la Red es que no se necesita un software de instalación adicional aparte del propio browser de WWW (Netscape, Mosaic, etc.), no hay passwords de entrada, el servicio es continuo, 24 horas al día durante 365 días al año, y la actualización de la información se realiza de forma totalmente transparente para el usuario. El coste de acceso a este tipo de información electrónica es mucho menor que el que pueda tener en soporte CD-ROM, ya que se parte de que la información en Internet se "alquila" no se "compra".

\section{Conclusiones}

Como conclusión, podemos decir que las fuentes de información electrónica tienen los siguientes beneficios para la investigación en cualquier área:

- El acercamiento de las fuentes hasta la propia mesa del investigador.

- Acceso a información, que en muchos casos sería de difícil consulta o localización, bien por reproducir obras de las que sólo existe un ejemplar, o por ser fuentes en mal estado de conservación y por lo tanto de difícil consulta.

Las herramientas informáticas permiten al investigador trabajar con los contenidos en formas y posibilidades mejores que las conocidas con la utilización de obras en papel. En este sentido son fundamentales los lenguajes de codificación, 
como SGML o HTML, que permitirán realizar búsquedas, combinaciones y comparaciones de versiones impensables hasta el momento.

Finalmente, las Bibliotecas han sido históricamente una de las fuentes primarias y fundamentales de información y por supuesto lo seguirán siendo en la era de la información electrónica, en que deben ser conscientes de que sus colecciones no se limitan al material ubicado en sus salas, sino que abarca, además, todos los recursos que los soportes electrónicos ponen a su disposición.

En este sentido, es fundamental el papel de las editoriales electrónicas cuyo trabajo ha sido y continuará siendo el de organizar los contenidos en un formato y reproducirlo en un soporte, ya sea éste papel, microficha, CD-ROM u On-Line.

En definitiva, la actividad, tanto de bibliotecas como editoriales electrónicas, debe encaminarse a la organización de datos, para que lleguen a ser Información, a partir de la cual se creen Herramientas de utilidad para la investigación.

\section{Referencias}

Baran, Nicholas (1995). Inside the Information Superhighway. Revolution. Scottsdale : Coriolis Group Books, 1995.

Currás, Emilia (1996). Tratado sobre ciencia de la información. Rosario : UNR Editora, 1996.

Martínez de Madariaga; Arroyo Fernández, D. (1994). Aproximación a Internet y su impacto en las bibliotecas y servicios de información. // Revista Española de Documentación Científica. 17 : 3 (1994) 277-289.

Tramullas Saz, Jesús (1996). Actas del Seminario Tendencias de Investigación en Documentación. Zaragoza : Universidad de Zaragoza, 1996. 